\title{
Streitkräfteeinsatz während der Coronakrise: Wie steht es um den OSZE-Verhaltenskodex?
}

\author{
Alexandre Lambert, Filip Ejdus, Thomas Schmidt"
}

\section{Kurzfassung}

Stand der Einsatz von Streitkräften während der Coronakrise in Europa im Einklang mit dem OSZE-Verhaltenskodex zu politisch-militärischen Aspekten der Sicherheit und anderen einschlägigen OSZE-Dokumenten? Dieser Beitrag zeigt, dass die meisten der von OSZE-Teilnehmerstaaten bislang als Reaktion auf die COVID-19-Pandemie ergriffenen Maßnahmen den OSZE-Normen und -Verpflichtungen entsprechen. Gleichzeitig offenbaren sich aber auch Schwachstellen. Dies gilt insbesondere für eine eindeutige Definition der Funktion und des Auftrags der Streit- und Sicherheitskräfte sowie für Vorkehrungen, die die Grundsätze der Notwendigkeit, Verhältnismäßigkeit und Nichtdiskriminierung beim Umgang mit öffentlichen Notlagen sichern sollen. In einigen wenigen Fällen wurde von Verpflichtungen im Zusammenhang mit Menschenrechten und Grundfreiheiten abgewichen. Wir formulieren Empfehlungen, um die Einhaltung der OSZE-Normen und -Verpflichtungen in Zukunft zu stärken, wenn es um den innerstaatlichen Einsatz von Streitkräften zur Unterstützung von Zivilbehörden in Ausnahmesituationen geht.

\section{Schlagworte}

Coronavirus, OSZE, Verhaltenskodex zu politisch-militärischen Aspekten der Sicherheit, Streitkräfte

Bitte zitieren als: Alexandre Lambert, Filip Ejdus, Thomas Schmidt, Streitkräfteeinsatz während der Coronakrise: Wie steht es um den OSZE-Verhaltenskodex?, OSCE Insights 5 (Baden-Baden: Nomos, 2021), https://doi.org/10.5771/9783748911630-05

\section{Einleitung ${ }^{1}$}

Gegen Ende 2019 tauchte in der Provinz Wuhan der Volksrepublik China eine

* Prof. Dr. Alexandre Lambert

SIT Study Abroad

alexandre.lambert@sit.edu

Prof. Dr. Filip Ejdus

Fakultät für Politikwissenschaft, Universität

Belgrad

filip.ejdus@fpn.bg.ac.rs atypische Lungenentzündung auf. Anfang 2020 wurde die Krankheit als COVID-19 identifiziert, verursacht durch das Coronavirus SARS-CoV-2. Am 30. Januar erklärte der Generalsekretär der Weltgesundheitsorganisation

(WHO)

\section{Oberst Thomas Schmidt}

Ständige Vertretung der Schweiz bei der OSZE, den Vereinten Nationen und anderen internationalen Organisationen in Wien thomas.schmidt@eda.admin.ch 
den Ausbruch der Krankheit zu einer gesundheitlichen Notlage von internationaler Tragweite. Bald darauf verbreitete sich das Virus schnell über die ganze Welt. Um diese beispiellose Notlage, die wir als Coronakrise kennen, einzudämmen, führten die meisten OSZE-Teilnehmerstaaten verschiedene Notstandsregelungen ein, zu denen auch der Einsatz von Streitkräften gehörte.

Moderne Streitkräfte sind große und gut organisierte Einrichtungen, die über eine umfangreiche Infrastruktur und bedeutende personelle wie materielle Ressourcen verfügen und sich daher in solchen Ausnahmefällen engagieren und die Zivilbehörden in Krisenzeiten unterstützen sollen. Dabei müssen sie jedoch nicht nur den verfassungsrechtlichen Rahmen, in dem sie sich bewegen, einhalten. Es gilt darüber hinaus, die internationalen Normen für die Anwendung von Gewalt, die unter anderem auf die OSZE zurückgehen, zu beachten. Als Sicherheitsorganisation befasst sich die OSZE zwar nicht direkt mit Fragen von Gesundheit und Medizin. Gleichwohl bietet der OSZE-Verhaltenskodex zu politischmilitärischen Aspekten der Sicherheit (im Folgenden „der Kodex“) von 1994 wichtige normative Leitlinien für den Einsatz von Streitkräften bei gesundheitlichen (öffentlichen) Notlagen, einschließlich gesundheitlichen Notlagen wie etwa der Coronakrise. ${ }^{2}$ Dies gilt umso mehr, als der Kodex sowohl für die politisch-militärische als auch die menschliche Dimension der Sicherheit von Bedeutung ist. ${ }^{3}$

Die zentrale Frage dieses Beitrags lautet, wie europäische Streitkräfte in der Coronakrise bislang eingesetzt wurden und ob dieser Einsatz den OSZE-Normen und -Verpflichtungen entspricht. Wir skizzieren zunächst die normativen Bestimmungen des Kodex und anderer OSZE-Dokumente, die gegebenenfalls auf den innerstaatlichen Gebrauch von Gewalt in gesundheitlichen Notlagen wie der Coronakrise anwendbar sind. Anschließend analysieren wir, wie unterschiedlich Staaten ihre Streitkräfte in der Krise eingesetzt haben. Dabei zeigen wir die wichtigsten Herausforderungen auf, die sich für die Einhaltung der einschlägigen OSZE-Normen ergeben. Wir stützen uns auf offizielle Dokumente der OSZE und der Teilnehmerstaaten, unter anderem die amtlichen Webseiten der Verteidigungsministerien und Streitkräfte, Länderberichte an die Europäische Organisation der Militärverbände und Gewerkschaften (EUROMIL), wissenschaftliche Arbeiten und Nachrichtenbeiträge. Unsere Analyse behandelt den Zeitraum ab der Anerkennung von COVID-19 als gesundheitliche Notlage im Januar 2020 bis Anfang September 2020, als der erste Höhepunkt der Pandemie in den meisten europäischen Ländern abgeklungen war und vor Einleitung der Maßnahmen im Hinblick auf die „Zweite Welle“. In ihrer Betrachtung umfasst die Analyse die europäischen OSZE-Teilnehmerstaaten. Sowohl innerstaatliche Einsätze als auch Ausnahmezustände bergen eindeutig Risiken. Die Analyse zeigt jedoch, dass die untersuchten Länder die OSZE-Normen im Allgemeinen eingehalten haben, wenngleich einige wenige bemerkenswerte Ausnahmen zu verzeichnen sind. Abschließend betrachten wir die Lehren aus 
der Coronakrise und sprechen politische Empfehlungen aus.

\section{Maßgebliche OSZE-Normen}

Die politisch bindenden OSZE-Dokumente enthalten mehrere Bestimmungen, die für den Einsatz von Streitkräften während der Coronakrise relevant sind. Ein erstes Paket an Bestimmungen findet sich in Dokumenten, die für die dritte, die menschliche Dimension der Sicherheit der OSZE von Bedeutung sind. Es bezieht sich auf öffentliche Notlagen im Allgemeinen. Das Moskauer Dokument von 1991 über die menschliche Dimension der Sicherheit (Ziff. 28.10) ${ }^{4}$ verpflichtet die Teilnehmerstaaten, die KSZE (Vorläuferin der OSZE) zu benachrichtigen, wenn ein Ausnahmezustand erklärt oder aufgehoben wird, und alle künftigen Abweichungen von ihren internationalen Menschenrechtsverpflichtungen aufgrund des Ausnahmezustands anzuzeigen. ${ }^{5}$ Das Schlussdokument des HelsinkiGipfels von $1992^{6}$ führt dies weiter aus. Dort ist festgelegt, dass das Büro für demokratische Institutionen und Menschenrechte (BDIMR/ODIHR) als Koordinierungsstelle der KSZE/OSZE für Informationen über einen öffentlichen Notstand dienen soll. Ziff. 24 und 25 des Kopenhagener Dokuments von 1990 sind ebenfalls von Bedeutung, wenn es um mögliche Abweichungen von Verpflichtungen mit Bezug zu Menschenrechten und Grundfreiheiten in öffentlichen Notlagen geht. $^{7}$ Sie verbieten eindeutig den Missbrauch und die willkürliche Anwendung von Einschränkungen von Men- schenrechten und Grundfreiheiten und legen fest, dass legitime Einschränkungen und Ausnahmen den Grundsatz der Verhältnismäßigkeit (Ziff. 24) zu wahren haben. Diese Bestimmung erinnert die Teilnehmerstaaten daran, dass es für bestimmte Menschenrechte keine Ausnahmeregelungen geben kann und diese daher selbst in Notlagen (oder Kriegszeiten) nicht eingeschränkt werden dürfen.

Schließlich enthält Ziff. 25 des Kopenhagener Dokuments spezifische Klauseln, in denen die Grundsätze der Öffentlichkeit (25.2), Notwendigkeit (25.3) und Nichtdiskriminierung (25.4) im Rahmen eines Notstands festgelegt sind. In Bezug auf die Notwendigkeit heißt es, dass „Maßnahmen, die Verpflichtungen außer Kraft setzen, auf den Umfang zu beschränken sind, den die Lage unbedingt erfordert". Zur Nichtdiskriminierung besagt Ziff. 25, dass „solche Maßnahmen eine Diskriminierung allein aufgrund der Rasse, der Hautfarbe, des Geschlechts, der Sprache, der Religion, der sozialen Herkunft oder der Zugehörigkeit zu einer Minderheit ausschließen werden" .8

Ein zweites Paket an Bestimmungen in OSZE-Dokumenten, die für die Coronakrise relevant sind, bezieht sich auf die Anwendung von Gewalt zu Zwecken der inneren Sicherheit. Wie bereits erwähnt, ist der Kodex die zentrale Quelle der OSZE für normative Bestimmungen zum Einsatz von Streitkräften. Er enthält ein Paket an ergänzenden Bestimmungen zum Einsatz von Streit- und Sicherheitskräften bei Aufgaben der inneren Sicherheit, die zum Beispiel für die Durchsetzung von Ausnahmezuständen gelten. Diese Bestimmungen legen 
fest, dass solche Aufgaben den internationalen Menschenrechtsstandards entsprechen müssen. Dazu gehören auch Standards in Bezug auf die Grundrechte und Grundfreiheiten von Angehörigen der Streitkräfte selbst. Sie ergänzen somit die oben genannten Normen der menschlichen Dimension.

Die für die Coronakrise besonders relevanten Artikel des Kodex (siehe Textbox) sind Art. 21 zur Bedeutung des verfassungsrechtlichen Rahmens für die demokratische Kontrolle, Art. 34 zur Achtung des Völkerrechts und Art. 36-37 mit Bestimmungen über den Einsatz von Streitkräften bei Aufgaben der inneren Sicherheit. Dazu gehört der oben bereits erwähnte Grundsatz der Verhältnismäßigkeit bei der (potenziellen) Anwendung von Gewalt.

\footnotetext{
\$21. Jeder Teilnehmerstaat wird jederzeit dafür sorgen und sicherstellen, daß seine militärischen und paramilitärischen Kräfte sowie seine Sicherheitskräfte durch die verfassungsgemäß errichteten und demokratisch legitimierten Organe wirksam geführt und kontrolliert werden. Jeder Teilnehmerstaat wird Vorkehrungen treffen, um zu gewährleisten, daß diese Organe ihre Verantwortlichkeiten nach Verfassung und Gesetz erfüllen. Sie werden die Funktionen und Aufgaben dieser Kräfte und deren Verpflichtung, ausschließlich im Rahmen der Verfassung zu handeln, eindeutig festlegen.

\$34. Jeder Teilnehmerstaat wird gewährleisten, daß seine Streitkräfte im Frieden und im Krieg so geführt, personell besetzt, ausgebildet und ausgerüstet werden, daß dies mit den Bestimmungen des Völkerrechts $[\ldots]$ im Einklang steht $[\ldots]$

\$36. Jeder Teilnehmerstaat wird gewährleisten, daß jeder Beschluß, seine Streitkräfte mit Aufgaben der inneren Sicherheit zu betrauen, im Einklang mit den verfassungsrechtlichen Verfahren gefaßt wird. Diese Beschlüsse werden den Streitkräften ihre Aufträge vorschreiben, wobei zu gewährleisten ist, daß diese unter der wirksamen Kontrolle durch verfassungsmäBige Organe sowie unter Einhaltung der Rechtsstaatlichkeit erfüllt werden. In Fällen, in denen zur Erfüllung von Aufgaben der inneren Sicherheit ein Rück-
}

griff auf Gewalt nicht vermieden werden kann, wird jeder Teilnehmerstaat gewährleisten, daß der Einsatz von Gewalt gegenüber den Erfordernissen der Durchsetzung nicht unverhältnismäßig sein darf. Die Streitkräfte werden gebührend dafür Sorge tragen, Schädigungen von Zivilpersonen oder von deren $\mathrm{Hab}$ und Gut zu vermeiden.

\$37. Die Teilnehmerstaaten werden Streitkräfte nicht dazu heranziehen, um die friedliche und gesetzmäßige Ausübung von Menschen- und Bürgerrechten durch Personen als Individuen oder Vertreter von Gruppen einzuschränken oder sie ihrer nationalen, religiösen, kulturellen, sprachlichen oder ethnischen Identität zu berauben.

Gemäß Ziff. 25.4 des Kopenhagener Dokuments, die den Grundsatz der Nichtdiskriminierung (siehe oben) behandelt, warnt Ziff. 17 des Kodex davor, dass Verletzungen von Menschenrechten und Grundfreiheiten zu politischen Spannungen führen können und dies Frieden und Sicherheit gefährden kann. Solche Verletzungen, heißt es dort, können „Erscheinungen von aggressivem Nationalismus, Rassismus, Chauvinismus, Fremdenfeindlichkeit und Antisemitismus" sein.

Schließlich enthält der Kodex spezifische Bestimmungen für Ausnahmezustände, die auf gesundheitliche Notlagen wie die Coronakrise anwendbar sind. Teilnehmerstaaten, die in einem Ausnahmezustand Streit- und Sicherheitskräfte einsetzen, müssen a) die Funktionen und Aufgaben der Streitkräfte und ihre Verpflichtung, ausschließlich im Rahmen der Verfassung zu handeln, eindeutig festlegen (Art. 21), b) gewährleisten, dass alle Angehörigen der militärischen und paramilitärischen Kräfte sowie der Sicherheitskräfte in der Lage sind, ihre Bürgerrechte wirksam auszuüben (Art.23, Art. 32), c) die politische Neutralität ihrer Streitkräfte als Ganzes wahren (Art. 23), 
d) den Schutz gegen den versehentlichen oder nicht autorisierten Gebrauch militärischer Mittel sicherstellen (Art. 24), e) die persönliche Verantwortlichkeit der Angehörigen der Streitkräfte nach dem innerstaatlichen und dem Völkerrecht gewährleisten (Art.30) undf) an dem Grundsatz festhalten, dass die Verantwortlichkeit der Vorgesetzten die Untergebenen von keiner ihrer persönlichen Verantwortlichkeiten - zum Beispiel gegenüber dem humanitären Völkerrecht und der Menschenrechtsgesetzgebung entbindet (Art. 31).

In Bezug auf die Anwendung des Kodex verpflichtet ein Beschluss des OSZEForums für Sicherheitskooperation die Teilnehmerstaaten, jährlich Informationen über die Umsetzung seiner Bestimmungen auszutauschen. ${ }^{9}$ Dazu gehört auch die freiwillige Berichterstattung über optionale Themen. Die Schweiz nutzte diese Gelegenheit im Rahmen des Informationsaustausches 2020 und fügte ihren Informationen ein kurzes Kapitel mit dem Titel „Maßnahmen zur Bekämpfung des Coronavirus“ hinzu. Darin betont sie unter anderem, dass alle nationalen Maßnahmen, die als Reaktion auf die Pandemie ergriffen wurden, im Einklang mit den Bestimmungen aus Ziff. 24 und Ziff. 25 des Kopenhagener Dokuments und Ziff. 28 des Moskauer Dokuments stehen. Darüber hinaus lud die Ständige Delegation der Schweiz bei der OSZE anlässlich der Jahresdiskussion über die Umsetzung des OSZE-Verhaltenskodex im Juni 2020 alle OSZE-Teilnehmerstaaten ein, den jährlichen Informationsaustausch als Gelegenheit zu nutzen, um gewonnene Erkenntnisse und bewährte
Praktiken im Zusammenhang mit dem Einsatz von Streitkräften in der Coronakrise zu teilen. ${ }^{10}$

\section{Ausnahmezustände und der Einsatz von Streitkräften während der COVID-19- Pandemie}

Seit Beginn der Coronakrise hat mehr als ein Drittel der OSZE-Teilnehmerstaaten offiziell den völkerrechtlich vorgesehenen öffentlichen Notstand ausgerufen. Andere Staaten haben sonstige, unterschiedlich strenge Notstandsregelungen eingeführt oder restriktive gesetzgeberische und politische Maßnahmen ergriffen. ${ }^{11}$ Achtundzwanzig Teilnehmerstaaten haben das ODIHR darüber informiert, dass sie als Reaktion auf die Pandemie Notfallmaßnahmen verabschiedet haben. ${ }^{12}$ Bei diesen Maßnahmen der Staaten ist es $\mathrm{zu}$ Abweichungen von Verpflichtungen mit Bezug zu Menschenrechten und Grundfreiheiten gekommen. Unter anderem betrifft dies die Versammlungs- und Vereinigungsfreiheit, die Freizügigkeit, das Recht auf Freiheit, das Recht auf ein faires Verfahren, das Recht auf Achtung des Privat- und Familienlebens, das Recht auf Sicherheit, Gedanken-, Gewissens- und Religionsfreiheit, das Recht auf Eigentum, das Recht auf Bildung und in einigen Fällen das Recht auf freie Meinungsäußerung sowie das Verbot von Folter und Misshandlung. Einige Länder, wie zum Beispiel Ungarn, führten einen unbefristeten Ausnahmezustand ohne die vom Kopenhagener Dokument geforderten zeitlichen Beschränkungen ein. ${ }^{13}$ Notfallmaßnahmen stellen 
darüber hinaus eine Herausforderung für die demokratische Kontrolle und die Funktionsweise der Parlamente, Transparenz, Privatsphäre (einschließlich des Schutzes personenbezogener Daten), die Justizinstitutionen, freie und faire Wahlen und die Bekämpfung von Hassverbrechen und Diskriminierung dar. ${ }^{14}$

In der gesamten OSZE-Region erleben die Teilnehmerstaaten eine Militarisierung des öffentlichen Diskurses eine Entwicklung, die in den meisten OSZE-Teilnehmerstaaten in jüngster Zeit ohne Beispiel ist. Hochrangige Politiker bemühen in ihren politischen Äußerungen vielfach Kriegsmetaphern. Sie sprechen in ihren öffentlichen Erklärungen häufig von Schlachten oder unsichtbaren Feinden und verwenden Analogien zu Krieg und militärischen Siegen. Diese Ausdrucksweise kann einerseits dazu beitragen, Dringlichkeit zu vermitteln, Lagerdenken auszusetzen, Solidarität zu fördern und Ressourcen zu mobilisieren, andererseits kann sie aber auch den Pluralismus schwächen, demokratische Verfahren untergraben und die internationale Solidarität behindern. ${ }^{15}$ Diese militaristische Sprache ebnet zudem den Weg sowohl für den gerechtfertigten als auch den ungerechtfertigten Einsatz von Streitkräften bei der Bewältigung der Krise.

In der Geschichte hat das Militär eine wesentliche Rolle bei der Eindämmung von Pandemien gespielt, von der Spanischen Grippe $1918^{16}$ bis zur H1N1-Pandemie 2009.17 Mit ihren unübertroffenen Fähigkeiten zur Krisenbewältigung, ihrer Forschungs- und Entwicklungsinfrastruktur, ihrer Disziplin, Autorität und Führungsstärke sind Streitkräfte ein natürlicher Partner für zivile Einrichtungen bei öffentlichen Notständen, auch im Bereich der öffentlichen Gesundheit. Es überrascht daher nicht, dass militärische Hilfe für zivile Einrichtungen in der gesamten OSZE-Region eine wesentliche Rolle bei der Coronakrise gespielt hat. In einigen Teilnehmerstaaten waren dies groß angelegte Operationen wie die Opération Résilience in Frankreich, die Operacion Balmis in Spanien und die Operation Restrict im Vereinigten Königreich. In den meisten Ländern erfolgte der Einsatz von Streitkräften „im Einklang mit den verfassungsrechtlichen Verfahren“ (Art. 36 des Kodex), es gab jedoch Ausnahmen. Sowohl in Serbien als auch in Albanien wurden beispielsweise die Parlamente umgangen, und die Exekutive unternahm keine Anstrengungen, zeitnah deren Zustimmung einzuholen. ${ }^{18}$

In der gesamten OSZE-Region wurde das Militär für verschiedene Aufgaben mobilisiert, die sich in fünf Hauptkategorien einteilen lassen (siehe Tabelle 1). 
Tabelle 1: Aufgaben, die während der Coronakrise von den Streitkräften der OSZETeilnehmerstaaten übernommen wurden

\begin{tabular}{|c|c|c|c|c|}
\hline $\begin{array}{l}\text { Logistik und } \\
\text { Transport }\end{array}$ & \begin{tabular}{|l} 
Medizinische \\
Unterstützung
\end{tabular} & $\begin{array}{l}\text { Forschung und } \\
\text { Entwicklung }\end{array}$ & $\begin{array}{l}\text { Unterstützung } \\
\text { bei der Regie- } \\
\text { rungsführung }\end{array}$ & $\begin{array}{l}\text { Innere } \\
\text { Sicherheit }\end{array}$ \\
\hline $\begin{array}{ll}\text { - } & \text { Rückho- } \\
\text { lung von } \\
\text { Staatsange- } \\
\text { hörigen } \\
\text { - } \text { Humanitäre } \\
\text { Hilfe } \\
\text { - Internatio- } \\
\text { nale Hilfe } \\
\text { - Transport } \\
\text { von Patien- } \\
\text { ten, medizi- } \\
\text { nischem } \\
\text { Personal } \\
\text { und PSA }\end{array}$ & $\begin{array}{ll}\text { - } \begin{array}{l}\text { Desinfekti- } \\
\text { on öffentli- } \\
\text { cher Berei- } \\
\text { che }\end{array} \\
\text { - Herstellung } \\
\text { von PSA } \\
\text { - Bau und Be- } \\
\text { trieb von } \\
\text { Feldlazaret- } \\
\text { ten - Tests } \\
\text { und Kon- } \\
\text { taktverfol- } \\
\text { gung } \\
\text { Bereitstel- } \\
\text { lung von } \\
\text { militäri- } \\
\text { schem Sani- } \\
\text { tätsperso- } \\
\text { nal, PSA, } \\
\text { Infrastruk- } \\
\text { tur und } \\
\text { Ausrüstung } \\
\text { Betrieb von } \\
\text { Leichenhal- } \\
\text { len }\end{array}$ & 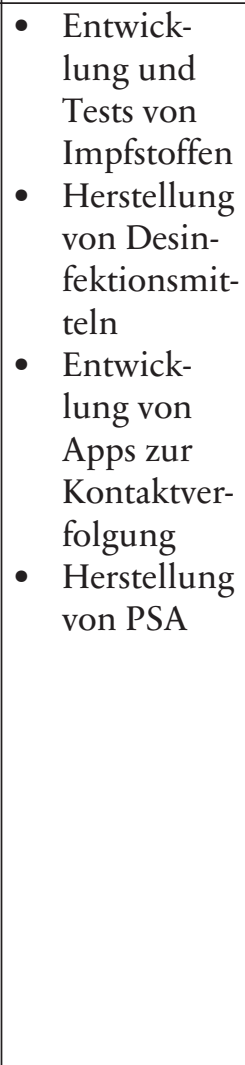 & 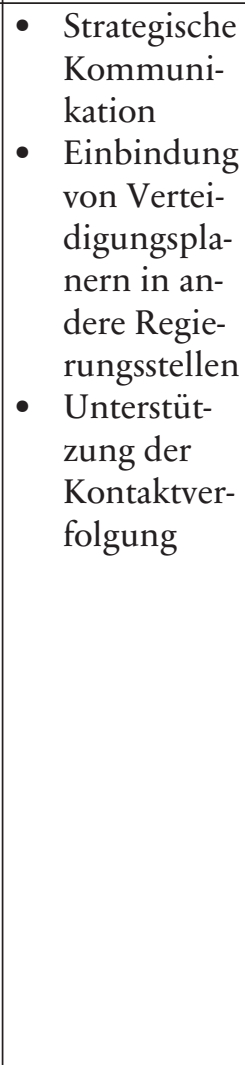 & $\begin{array}{ll}\text { - } & \text { Grenzkon- } \\
\text { trolle } \\
\text { - } \\
\text { Migrations- } \\
\text { manage- } \\
\text { ment } \\
\text { - Gewährleis- } \\
\text { tung von } \\
\text { Recht und } \\
\text { Ordnung } \\
\text { - Durchset- } \\
\text { zung der } \\
\text { Quarantäne } \\
\text { Schutz von } \\
\text { Kranken- } \\
\text { häusern, Se- } \\
\text { niorenzen- } \\
\text { tren, öffent- } \\
\text { lichen Un- } \\
\text { ternehmen } \\
\text { und kriti- } \\
\text { scher Infra- } \\
\text { struktur }\end{array}$ \\
\hline
\end{tabular}

Erstens setzten Teilnehmerstaaten ihre Streitkräfte für Logistik und Transport ein. Dazu gehören die Rückholung von Staatsangehörigen, die als Touristen oder Arbeitskräfte im Ausland gestrandet waren, und die humanitäre Hilfe im Land. So verteilten sie Lebensmittel in Albanien an in Armut lebende Menschen, ${ }^{19}$ in
Malta an Menschen in Quarantäne ${ }^{20}$ und in Belgien an Obdachlose. ${ }^{21}$ Darüber hinaus beteiligte sich das Militär an der Versendung und am Empfang internationaler Hilfe, unter anderem am Transport von Patienten, medizinischem Personal und persönlicher Schutzausrüstung (PSA). Diese militärischen Logistik- und 
Transportaktivitäten waren unumstritten und verstießen nicht gegen den Kodex oder andere zentrale Dokumente.

Zweitens setzten Teilnehmerstaaten ihre Streitkräfte zur medizinischen Unterstützung ein, unter anderem für die Desinfektion öffentlicher Bereiche, die Herstellung von PSA, den Bau und Betrieb von Feldlazaretten, Tests und Kontaktverfolgung, die Bereitstellung von militärischem Sanitätspersonal, PSA, Infrastruktur und Ausrüstung sowie den Betrieb von Leichenhallen. Diese Aktivitäten sind zwar verhältnismäßig unumstritten, in Bezug auf den Kodex jedoch nicht unproblematisch. Zum einen handelt es sich dabei um Aktivitäten, die Soldat*innen potenziell dem Virus aussetzen und Fragen nach den Menschenrechten und Grundfreiheiten der Militärangehörigen, insbesondere hinsichtlich ihrer Gesundheit und Sicherheit am Arbeitsplatz, aufwerfen (Art. 32 des Kodex). In vielen Fällen wurde (in der Regel von Militärgewerkschaften) berichtet, dass Soldat*innen keine angemessene Ausbildung oder ausreichende PSA hatten, was zu unnötigen Infektionen und Todesfällen (beim Personal) führte. ${ }^{22}$ Darüber hinaus ist die Mithilfe des Militärs beim Testen der Bevölkerung und der Kontaktverfolgung bedenklich, da hier Menschenrechtsverletzungen und Spannungen zwischen dem zivilen und dem militärischen Sektor möglich sind (Art. 2, Art. 17 des Kodexes). In der Slowakei führten beispielsweise die Streitkräfte die obligatorischen Tests der Roma-Bevölkerung durch, was laut Amnesty International die „Stigmatisierung und die Vorurteile, denen sie be- reits ausgesetzt ist“, nur noch verschärft hat. ${ }^{23}$

Drittens wurden die Streitkräfte für Forschung und Entwicklung eingesetzt. Dazu gehören die Entwicklung und das Testen von Impfstoffen, Desinfektionsmitteln, Apps zur Kontaktverfolgung und PSA. Die einzige in der vorliegenden Studie ausgewiesene Aktivitat in dieser Kategorie, die moglicherweise gegen den Kodex (Art. 32) verstößt, ist das Testen von Impfstoffen an russischen Soldaten. ${ }^{24}$ Auch wenn sich die Soldaten freiwillig gemeldet haben, ist dies insofern potenziell problematisch, als dass das Militär eine hierarchische Struktur ist, in der Druck, entweder direkt oder indirekt, den Grundsatz der Einwilligung nach vorheriger Aufklärung aushebeln kann. ${ }^{25}$ Andere bekannte Forschungs- und Entwicklungsaktivitäten, die von den Streitkräften der OSZE-Teilnehmerstaaten durchgeführt wurden, waren im Hinblick auf die Einhaltung des Kodex unproblematisch.

Viertens setzten Teilnehmerstaaten ihre Streitkräfte zur Unterstützung bei der Regierungsführung für andere Regierungsorgane ein. In Litauen ${ }^{26}$ unterstützten zum Beispiel Militärexperten für strategische Kommunikation die zivilen Institutionen bei der Eindämmung von Desinformation und Fake News. ${ }^{27}$ Die britischen Streitkräfte entsandten Militärplaner in Kommunalverwaltungen, um bei Planung und Entscheidungsfindung behilflich zu sein. ${ }^{28}$ In Irland unterstützten Angehörige der Verteidigungskräfte die Gesundheitsbehörden bei der Ermittlung von Kontaktpersonen. ${ }^{29}$ Planer der niederländischen Streitkräfte wurden den 
nationalen Krisenstrukturen, dem Gesundheitsministerium und anderen zivilen Institutionen zugeteilt, um ihnen bei der Koordination zu helfen. ${ }^{30}$ Die Unterstützung ziviler Behörden durch die Streitkräfte in diesen und ähnlichen Funktionen ist unproblematisch, solange deren Einsatz "unter der wirksamen Kontrolle durch verfassungsmäßige Organe sowie unter Einhaltung der Rechtsstaatlichkeit" (Art. 36) erfolgt und solange sie als Ganzes politisch neutral bleibt (Art. 23). In allen Fällen, die den Verfassern dieses Beitrags bekannt sind, wurde diese Bestimmung eingehalten, und in der beobachteten Region wurden keine Fälle festgestellt, in denen militärische Gremien Entscheidungen getroffen haben, die keiner zivilen demokratischen Kontrolle unterlagen.

Schließlich setzte eine beträchtliche Anzahl von Teilnehmerstaaten ihre Streitkräfte für Aufgaben der inneren Sicherheit ein. Dazu gehören Grenzkontrollen, Migrationsmanagement, die Gewährleistung von Recht und Ordnung, die Durchsetzung von Quarantäne und der Schutz von Krankenhäusern, Seniorenzentren, öffentlichen Unternehmen und kritischer Infrastruktur. Einige Länder, wie Deutschland und Großbritannien, verzichteten aufgrund verfassungsrechtlicher Zwänge und ihrer geschichtlichen Erfahrungen auf einen solchen Einsatz ihrer Streitkräfte. ${ }^{31}$ Aus mehreren Teilnehmerstaaten, in denen dem Militär eine solche Rolle übertragen wurde, wurde von unangemessener Gewaltanwendung berichtet. In Serbien wurde zum Beispiel die Militärpolizei mobilisiert, um die Asylzentren zu bewa- chen, in denen die rund 9.000 Flüchtlinge im Land während des Ausnahmezustands festgehalten wurden. ${ }^{32}$ Aus Irland und den Niederlanden wurde ebenfalls berichtet, Asylsuchende seien auf Militärgelände interniert worden. ${ }^{33}$ Das Militär wurde in mehreren OSZE-Teilnehmerstaaten auch zum Schutz der Grenzen eingesetzt, darunter Griechenland, Kroatien, Polen, die Tschechische Republik, Lettland, Litauen, die Niederlande, Nordmazedonien, Österreich, Portugal, Serbien, die Slowakei und Slowenien. Dabei waren Streitkräfte einiger Teilnehmerstaaten an der Zurückdrängung von Migranten und an Menschenrechtsverletzungen beteiligt. In Kroatien war das Militär in eine gewaltsame Zurückdrängungsaktion an der Landesgrenze verwickelt, während in Slowenien paramilitärische Bürgerwehren an der Südgrenze des Landes patrouillierten, um Migranten an der Einreise zu hindern. ${ }^{34}$ In vielen Teilnehmerstaaten wurden Streitkräfte eingesetzt, um die Einhaltung der Quarantänevorschriften zu gewährleisten, auf den Straßen zu patrouillieren und Einrichtungen zu bewachen. Eine solche militärische Hilfe für zivile Einrichtungen ist in bestimmten Kontexten akzeptabel, insbesondere, wenn die Kapazitäten der Polizei erschöpft sind. Besorgniserregend war jedoch, dass es in mehreren Fällen keine klar formulierte Begründung oder Definition der Funktionen und Aufgaben der Streitkräfte gab. ${ }^{35}$ In Ungarn ${ }^{36}$ und Serbien $^{37}$ war der massive Einsatz des Militärs für Zwecke der inneren Sicherheit nicht immer durch Erwägungen der öffentlichen Gesundheit gestützt. Der anhaltende demokratische Rückschritt, der in die- 
sen Ländern stattgefunden hat, wurde dadurch nur noch verschlimmert.

\section{Fazit und politische Empfehlungen}

Die COVID-19-Pandemie hat die Welt kalt erwischt. Sie hat Schwächen in den Fähigkeiten nationaler Regierungen und internationaler Organisationen aufgedeckt, ihre Bemühungen zur rechtzeitigen Eindämmung der Ausbreitung des Virus zu koordinieren und ihre Mittel verhältnismäßig und so einzusetzen, dass die Menschenrechte und Grundfreiheiten ihrer Bürger konsequent gewahrt werden. Mehr als ein Drittel der Teilnehmerstaaten riefen den Ausnahmezustand aus, und einige wandten ungewöhnlich drakonische, maximal restriktive LockdownMaßnahmen an. Im gesamten europäischen Teil der OSZE-Region setzten Länder Streitkräfte für den Transport, die medizinische Unterstützung, für Forschung und Entwicklung, die Unterstützung der Regierungsführung und die Gewährleistung der inneren Sicherheit ein.

Der Streitkräfteeinsatz war in den meisten Fällen gerechtfertigt und unumstritten, in einigen Fällen, insbesondere wenn es um die Gewährleistung der inneren Sicherheit ging, stand er jedoch nicht im Einklang mit dem OSZE-Verhaltenskodex. Die Aktivitäten der Streitkräfte in modernen, entwickelten Staaten müssen sich auf Rechtsstaatlichkeit und einen stabilen, klar definierten Rechtsrahmen stützen. Streitkräfte sind strategische Reserven und "harte Machtfaktoren“, auf die Staaten zurückgreifen können, um sich legitim gegen äußere Feinde zu verteidigen und im Rahmen von Mandaten des Sicherheitsrats der Vereinten Nationen für Friedenserzwingung und Friedenserhaltung zu sorgen. Streitkräfte sind daher strukturell schlecht geeignet, nichtmilitärische innenpolitische Probleme wie die Coronakrise anzugehen, wie die skizzierten Beispiele für Fehlverhalten und Kompetenzüberschreitung zeigen.

Der Einsatz von Streitkräften in außerordentlichen Situationen und Ausnahmezuständen und der Grad angemessener parlamentarischer und demokratischer Kontrolle solcher Einsätze ist ein wichtiger Lackmustest dafür, wie engagiert eine Gesellschaft (und ihre Spitzenpolitiker) für demokratische Standards eintritt. Unzulänglichkeiten beim Einsatz von Streitkräften während der COVID-19-Krise sind somit Symptome grundlegender tiefwurzelnder, systemischer Probleme in der Regierungsführung. Wenn diese Versäumnisse auf lediglich schlechte Umsetzung, Stress und mangelnde Erfahrung zurückgeführt werden können, lassen sich Unzulänglichkeiten und Fehler in der Zukunft dadurch vermeiden, dass die Vertreter aus Politik und Parlament die Fakten gründlich bewerten und aus den Erfahrungen lernen. Wenn sie jedoch aus einem allgemeinen Versagen der Regierungsführung entstanden sind, ist dies auf schwache demokratische Institutionen und/oder eine schwache demokratische politische Kultur zurückzuführen. Die Coronakrise kann daher als aufschlussreicher Indikator für den Staat und die Qualität der demokratischen Regierungsführung in der gesamten Region dienen. Dabei sei auch auf Art. 20 des Kodex und insbesondere auf seine Schluss- 
bestimmung verwiesen, wonach die Teilnehmerstaaten „[...] die Integration ihrer Streitkräfte in die zivile Gesellschaft als wichtigen Ausdruck der Demokratie fördern" werden.

Analysiert man die jüngsten Einsätze von Streitkräften als Reaktion auf die Coronakrise vor dem Hintergrund der im Kodex verankerten Normen und Grundsätze, so lassen sich einige vorläufige politische Empfehlungen formulieren, die die OSZE und ihre Teilnehmerstaaten berücksichtigen sollten:

- Notfallmaßnahmen und der Einsatz von Streitkräften müssen innerhalb des verfassungsrechtlichen Rahmens der Staaten und unter voller demokratischer, parlamentarischer und ziviler Kontrolle erfolgen. Die OSZE-Teilnehmerstaaten sollten in diesem $\mathrm{Zu}$ sammenhang die einschlägigen Normen und Bestimmungen des Kopenhagener Dokuments von 1990, des Moskauer Dokuments von 1991 über die menschliche Dimension der Sicherheit, des Abschließenden Dokuments des Gipfeltreffens von Helsinki von 1992 und des OSZE-Verhaltenskodex zu politisch-militärischen Aspekten der Sicherheit von 1994 anwenden.

- COVID-19-Task Forces sollten Ad-hocEinrichtungen der Regierung sein, die von Regierungschefs oder Gesundheitsministern und nicht von Verteidigungsministerien geführt werden. Wenn Streitkräfte den zivilen Institutionen im Zusammenhang mit einer Pandemie oder einer anderen zivilen Aufgabe militärische Hilfe leisten, sollten sie eine streng untergeordnete Rolle spielen und der leitenden Zivilbehörde unterstellt sein.

- Kompetente und klare Hierarchien sowie maßgeschneiderte Verhaltensregeln (rules of behaviour - ROB) und/ oder Einsatzregeln (rules of engagement - ROE) sind von größter Bedeutung, damit Streitkräfte im zivilen Umfeld effizient (und verfassungskonform) eingesetzt werden und Streitkräfte und Zivilisten erfolgreich zusammenwirken können.

- Gemeinsame Schulungen und Übungen aller Institutionen und Behörden, die sich mit Notsituationen und Katastrophenhilfe befassen, sollten regelmäßig auf nationaler, regionaler und lokaler Ebene durchgeführt werden, um so die Effizienz und Wirksamkeit ihrer Maßnahmen zu erhöhen. Diese verschiedenen Regierungsebenen sollten auch ein gemeinsames Verständnis dafür entwickeln, wie sie miteinander agieren. Die jeweiligen Aufgaben werden dabei in einer Liste nach dem Subsidiaritätsprinzip eindeutig zugewiesen.

- Angehörige der Streitkräfte, die während der Pandemie eingesetzt werden, sollten so geschult und ausgerüstet werden, dass die Ansteckungsgefahr minimiert wird. Militärische Aktivitäten, die für die nationale Sicherheit oder die Landesverteidigung (aktuelle Konflikte, Verteidigung gegen bewaffnete Angriffe usw.) unwesentlich sind oder bei denen die gesundheitlichen Vorkehrungen nicht vollständig umgesetzt werden können, sollten ausgesetzt oder zurückgefahren werden. 
- Im Rahmen der dienstlichen Erfordernisse sollten die Militärangehörigen in der Lage sein, ihre Menschenrechte und Grundfreiheiten wahrzunehmen und auszuüben. Dazu gehört ihre garantierte individuelle körperliche Unversehrtheit. Militärangehörige sollten nicht unnötig biologischen Gefahren ausgesetzt werden; wenn sie es dennoch sind, sollten sie ausreichend geschützt sein. Darüber hinaus sollte den Angehörigen der Streitkräfte nicht befohlen werden, sich ,freiwillig“ zu melden, um neu entwickelte Impfstoffe oder Medikamente zu testen. Dies wäre ein klarer Missbrauch der militärischen Hierarchie.

- Die Teilnehmerstaaten sollten den jährlichen Informationsaustausch zum Kodex, der als Teil des VSBM-Instrumentariums der OSZE betrachtet wird, verstärkt als Plattform nutzen, um sich darüber auszutauschen, wie sie mit der durch die Pandemie verursachten Notsituation umgehen und die Normen und Bestimmungen des Kodex einhalten. ${ }^{38}$

- Die OSZE-Teilnehmerstaaten wie auch das OSZE-Sekretariat sollten sich verstärkt darum bemühen, das Bewusstsein für die Bedeutung des Kodex zu schärfen. In den letzten Jahren hat die Bedeutung der OSZE in den Augen vieler Teilnehmerstaaten leider abgenommen. Viele OSZE-Dokumente sind nicht mehr hinreichend bekannt, so dass Regierungen gegebenenfalls einen Notfalleinsatz von Truppen planen, ohne den Kodex als zentrales normsetzendes Dokument zu konsultieren.
- Seminare, Workshops und andere Aktivitäten in der gesamten OSZE-Region, die die Teilnehmerstaaten umfassender mit dem Kodex vertraut machen, sollten weiter gefördert und intensiviert werden. Darüber hinaus könnte eine überarbeitete Fassung des Kompendiums praktischer Beispiele für die demokratische Kontrolle von Streitkräften, eines Leitfadens für bewährte Praktiken bei der Umsetzung des Verhaltenskodex in der gesamten OSZE-Region, ein Kapitel aufnehmen, das sich mit der Frage der Umsetzung des Kodex in Ausnahmesituationen/Notlagen befasst. ${ }^{39}$

- Notfallmaßnahmen und die Herausforderung, sie verhältnismäßig einzusetzen, sind (wie die Coronakrise gezeigt hat) nicht neu; sie spiegeln generellere Unzulänglichkeiten wider, insbesondere in Ländern mit schon bestehenden Schwierigkeiten und Mängeln in der Regierungsführung. Einige Teilnehmerstaaten haben bereits angekündigt, ihre Reaktion auf COVID-19 evaluieren zu wollen. Dabei sollte das Handeln aller Exekutivorgane, sowohl der zivilen als auch der militärischen, und aller Akteure von der obersten Kommando- und Kontrollebene bis hinunter zu Polizei und Militär vor Ort beurteilt werden. Ziel sollte es sein festzustellen, inwieweit diese Akteure die Grundsätze der Verhältnismäßigkeit und des geringstmöglichen Schadens eingehalten haben.

- Das ODIHR könnte die Teilnehmerstaaten bei der Durchführung solcher Beurteilungen und Selbstevaluie- 
rungen unterstützen und sich dabei auf allgemeine Konzepte und Erfahrungen aus früheren Fällen stützen. Im Anschluss an seine Beurteilung der Lage in der OSZE-Region könnte das ODIHR allgemeine Empfehlungen an alle Teilnehmerstaaten aussprechen, indem es einen Praxisleitfaden zur parlamentarischen demokratischen Kontrolle der Streitkräfte in Ausnahmezuständen und Notlagen veröffentlicht. Darüber hinaus sollte das ODIHR die Möglichkeit haben, die Schlussfolgerungen seiner OSZEweiten Beurteilung mitzuteilen, indem es den einzelnen Teilnehmerstaaten auf Wunsch maßgeschneiderte Empfehlungen gibt.

\section{Notizen}

1 Die Autoren bedanken sich bei den anonymen Gutachtern für ihre wertvollen Kommentare zu einer früheren Version dieses Manuskripts.

2 OSZE, Verhaltenskodex zu politisch-militärischen Aspekten der Sicherheit, DOC.FSC/1/95, 3. Dezember 1994, https: //www.osce.org/de/fsc/41357.

3 Prasenjit Chaudhuri, Alexandre Lambert and Thomas Schmidt (eds.), „20 years of OSCE Code of Conduct on Politico-Military Aspects of Security," Swiss Armed Forces, June 2015, https:/www.osce.org/f iles/f/documents/8/4/128961.pdf.

4 OSZE, Dokument des Moskauer Treffens der Konferenz über die menschliche Dimension der KSZE vom 3. Oktober 1991, https://www.osce.org/odihr/electio ns/14310.

5 Die Konferenz über Sicherheit und Zusammenarbeit in Europa (KSZE) wurde 1995 in Organisation für Sicherheit und
Zusammenarbeit in Europa (OSZE) umbenannt.

6 OSZE, KSZE Helsinki-Dokument 1992: Herausforderung des Wandels, 9.-10. Juli 1992, https://www.osce.org/de/mc/39532.

7 OSZE, Dokument des Kopenhagener Treffens der Konferenz über die menschliche Dimension der KSZE, 29. Juni 1990, https://www.osce.org/odihr/electio ns/14304.

8 Weitere internationale Normen und Bestimmungen, die für OSZE-Teilnehmerstaaten relevant sind, sind im Internationalen Pakt über bürgerliche und politische Rechte (Art. 12, 17, 21), in der Europäischen Konvention zum Schutze der Menschenrechte und Grundfreiheiten (Art. 8, 11) und in Art. 2 des Protokolls Nr. 4 zur Europäischen Konvention niedergelegt.

9 OSZE, Forum für Sicherheitskooperation, Beschluss Nr. 2/09: Technisches Update des Fragebogens zum Verhaltenskodex, FSC.DEC/2/09, 1. April 2009, https:/ /www.osce.org/fsc/36748.

10 Siehe: Permanent Mission of Switzerland to the Organization for Security and Cooperation in Europe (OSCE), the United Nations and to the International Organizations in Vienna, Information Exchange on the Code of Conduct on Politico-Military Aspects of Security, Note 21/2020-OSZE, FSC.EMI/75/20, 15 April 2020, S. 18, https://www.osce.org/files/f/d ocuments/8/3/450214.pdf.

11 Gemäß Veröffentlichung des ODIHR-Berichts: OSCE/ODIHR, OSCE Human Dimension Commitments and State Responses to the Covid-19 Pandemic, 2020, S. 20, https://www.osce.org/odihr/human -rights-states-of-emergency-covid19.

12 OSCE/ODIHR, Ebd., S. 11.

13 OSCE/ODIHR, Ebd., S. 36.

14 OSCE/ODIHR, Ebd., S. $64 \mathrm{f}$.

15 Eric Van Rythoven, „What's wrong with the war metaphor", Duck of Minerva, 5 
April 2020, https://duckofminerva.com/2 020/04/whats-wrong-with-the-war-metap hor.html.

16 Christopher Watterson und Adam Kamradt-Scott, „Fighting Flu: Securitization and the Military Role in Combating Influenza“, Armed Forces \& Society 1/2016, 145-168.

17 Hazan V. Molina et al, „The Military Role in a Flu Pandemic", Harefuah 1/2010, S. 9.

18 Katarina Đokić, „The armed forces against COVID-19 in the Western Balkans: One invisible enemy and a few visible flaws", European Western Balkans, 5 May 2020, https://europeanwesternbalka ns.com/2020/05/05/the-armed-forces-agai nst-covid-19-in-the-western-balkans-one-i nvisible-enemy-and-a-few-visible-flaws/.

19 Republik Albanien, Streitkräfte, „COVID - 19, FA vijojnë angazhimin në operacionin humanitar dhe në monitorim/ patrullim në gjithë vendin“, 15. April 2020, https://aaf.mil.al/te-fundit/5514-cov id-19-fa-vijojne-angazhimin-ne-operacion in-humanitar-dhe-ne-monitorim-patrulli m-ne-gjithe-vendin.

20 "Soldiers delivering groceries to people in quarantine, CPD assisting police in spot checks“, Malta Independent, 16 March 2020, https://www.independent. com.mt/articles/2020-03-16/local-news/So ldiers-delivering-groceries-to-people-in-qu arantine-CPD-assisting-police-in-spot-che cks-6736220894.

21 "CORONA - What are the Belgian Armed Forces doing in the fight against Covid-19?"“, EUROMIL, March 2020, http://euromil.org/wp-content/uploads /2020/03/Corona-What-are-the-Belgian-A rmed-Forces-doing.pdf.

22 Siehe zum Beispiel den Bericht der United Association of Spanish Military, „INFORME Situación del COVID-19 en Fuerzas Armadas ¿QUIÉN PROTEJE AL QUE PROTEJE?“, EUROMIL, 2 April 2020, http://euromil.org/wp-content/upl
oads/2020/04/200402-Informe-AUME-pre vención-COVID-19.pdf.

23 Amnesty International, „Roma must not be further stigmatized during COVID-19“, EUR 72/2110/2020, 8 April 2020, https://www.amnesty.org/downloa d/Documents/EUR7221102020ENGLIS H.PDF.

24 „Russia's coronavirus vaccine 'ready', military says", The Moscow Times, 21 July 2020, https://www.themoscowtimes.co $\mathrm{m} / 2020 / 07 / 21 /$ russias-coronavirus-vaccine -ready-military-says-a70941.

25 „Russia's coronavirus vaccine trials have a few problems", Meduza, 5 June 2020, https://meduza.io/en/feature/2020/06/06/ russia-s-coronavirus-vaccine-trials-have-a-f ew-key-problems.

26 Republic of Lithuania, Ministry of $\mathrm{Na}$ tional Defence, „Frequently asked questions about the Lithuanian Armed Forces in the context of COVID19“, 14 April 2020, https://kam.lt/en/news_1098/curre nt_issues/frequently_asked_questions_ab out_the_lithuanian_armed_forces_and_c ovid19.html.

27 „MOD team to tackle coronavirus fake news and scams", Forces, 9 April 2020, https://www.forces.net/news/mod-team-t ackle-coronavirus-fake-news-and-scams.

28 Erica Pepe/Amanda Lapo, „Europe's armed forces and the fight against COVID-19“, IISS, 9 April 2020, https:// www.iiss.org/blogs/military-balance/2020 /04/europe-armed-forces-covid-19.

29 „80 Defence Forces cadets are being trained in Covid-19 contact tracing“, The Journal, 13 March 2020, https://www.the journal.ie/defence-forces-cadets-trained-i $\mathrm{n}$-coronavirus-contact-tracing-5046020-M ar2020/.

30 Regierung der Niederlande, Verteidigungsministerium, „Deployment of the Netherlands Ministry of Defence in the Fight against COVID-19, 11 March-1 July“, https://english.defensie.nl/topics/cor ona-covid-19. 
31 Pepe und Lapo, oben zitiert (Anm. 28).

32 Nikola Kovačević, Deprivation of liberty of refugees, asylum seekers and migrants in the Republic of Serbia through measures of restriction and measures of derogation from human and minority rights made under the auspices of the state of emergency, A 11 - Initiative for Economic and Social Rights, 16 March 2020, https://www.a11initiative.org/wp-content /uploads/2020/05/Deprivation-of-liberty-o f-refugees.pdf.

33 Mark Akkerman, „COVID-19 and Border Politics“, Border Wars Briefing 1, Transnational Institute/Stop Wapenhandel, July 2020, https://www.tni.org/files/public ation-downloads/tni-covid-19-and-border -politics-brief.pdf.

34 Dušan Stojanović, „Vigilantes in Slovenia patrol borders to keep out migrants“, AP,
17. September 2019, https://apnews.com/ $57424 \mathrm{e} 6 \mathrm{bf} 60046 \mathrm{e} 594 \mathrm{~b} 4 \mathrm{c} 052 \mathrm{bac} 86 \mathrm{~b} 6 \mathrm{c}$.

35 Akkerman, oben zitiert (Anm. 33).

36 BIRN, „Hungarian 'militarisation' under Orban stirs concern", Reporting Democracy, 29 July 2020, https://balkaninsight.c om/2020/07/29/hungarian-militarisation -under-orban-stirs-concern/.

37 Filip Ejdus, „Pandemijske lekcije za politiku i system odbrane Republike Srbije“, MONS, 7 May 2020, http://mons.rs/pand emijske-lekcije-za-politiku-i-sistem-odbra ne-republike-srbije.

38 Siehe auch Anm. 10 dieses Berichts.

39 Annual discussion on the implementation of the Code of Conduct on politicomilitary aspects of security, Consolidated Report, FSC.GAL/96/14, 24 July 2014, http://www.osce.org/fsc/121796. 
